\title{
Analyses of the anode region of a Hall thruster channel
}

\author{
Michael Keidar, Iain D. Boyd, \\ Department of Aerospace Engineering, University of Michigan, Ann Arbor MI 48109 \\ Isak I. Beilis \\ Electrical Discharge and Plasma Laboratory, Tel Aviv University, Israel
}

\begin{abstract}
We present analyses of the anode region in a Hall thruster. In the case when the existence of an anode sheath is essential the ion backflow region (presheath) is considered. Ion acceleration in the near anode region occurs in a two-dimensional manner in order to satisfy the requirement for two presheaths: one near the anode and one near the lateral wall (dielectric). It is shown that the length of the anode presheath is affected significantly by the magnetic field and the mass flow rate. It is concluded that the sonic transition in the Hall thruster should have a two-dimensional structure.
\end{abstract}




\section{Introduction}

A plasma thruster with closed electron drift or so-called Hall thruster is currently one of the most advanced and efficient types of electrostatic propulsion devices. The Hall thruster produces much higher thrust density than other types of stationary ion thrusters. This advantage comes from the fact that acceleration takes place in a quasi-neutral plasma and thus is not limited by space charge effects. State of the art Hall thrusters have an efficiency of about $50 \%$ with specific impulses in the range of $1000 \mathrm{~s}-3000 \mathrm{~s} .^{1,2,3}$ The electrical discharge in the Hall thruster has a cross-field (E×B) configuration where the external magnetic field is radial and perpendicular to the axial electric field, which accelerates the ions. The electron current passing across the magnetic field leads to an electron closed drift or Hall drift. Generally, two different types of Hall thruster were developed: a thruster with closed electron drift and extended acceleration zone, or Stationary Plasma Thruster (SPT), and a thruster with short acceleration channel or Thruster with Anode Layer (TAL). In this paper we will consider the first type of thruster, namely SPT, which employs a dielectric channel that plays an important role in the discharge.

It was recognized that in a SPT, the interaction of the plasma with the dielectric wall plays an important role. The whole idea of introducing the dielectric wall into the discharge chamber was in order to actively affect plasma parameters. Due to the collisions of the electrons with the wall and secondary electron emission, the electron temperature remains relatively low in comparison to the TAL. As a result, the ion acceleration occurs over a more extended region. ${ }^{4}$ Despite many theoretical efforts, the complicated picture of the physical processes in the Hall thruster channel are far from being completely understood. Mainly the physics of the plasma interaction with a dielectric wall and the anode, as well as transition between the quasi-neutral plasma and the sheath have not been investigated in detail. However, experimental efforts in this direction are on the way. 
For instance it was found that the dielectric material substantially affects the discharge behavior in the Hall thruster ${ }^{5}$. More recently it was found that use of sectioned electrodes inside the Hall thruster channel with different secondary electron emission properties have a considerable effect on the discharge characteristics as well as on thruster performance ${ }^{6}$. These findings suggest that the effect of the plasma interaction with a dielectric wall is an important issue in Hall thrusters.

The transition plasma-wall region determines the particle and energy fluxes from the plasma to the wall. Recently we presented a model of plasma wall transition that accounts for secondary electron emission $^{7}$. In order to develop a self-consistent model, the boundary parameters at the sheath edge (ion velocity and electric field) are obtained from a two-dimensional plasma bulk model. In the considered condition, i.e. ion temperature much smaller than that of electrons and significant ion acceleration in the axial direction, the presheath scale length becomes comparable to the channel width so that the plasma channel becomes an effective presheath. Additionally it was shown that a modified Bohm criteria is more appropriate for describing the plasma wall interaction. It was shown that a plasma-sheath matching approach proposed previously can be used. ${ }^{8}$ In this approach, the electric field that develops in the presheath can serve as a boundary condition for the sheath. At the same time the model predicts that the quasi-neutrality assumption at the presheath edge is still valid.

Our previous model $^{7}$ considers mainly the acceleration channel after the sonic transition, since we expected the main effect of the dielectric wall (i.e. secondary electron emission) to lie in this region. However, sonic transition for the ion flow has an important role ${ }^{9}$. It is therefore important to consider the diffuse region from the anode to the sonic transition plane. In the usual situation, the electron thermal current in the near anode sheath is larger than the discharge current and therefore a negative anode sheath is present. As a result, there is a requirement for ion flow to the anode. Ion velocity at the anode sheath edge should satisfy sheath criteria. This condition was used as a 
boundary condition for the plasma flow problem in the Hall thruster channel. ${ }^{10}$ It should be noted that there is a possibility of solution of the Hall thruster flow without anode sheath as was suggested recently. ${ }^{11}$ Considering the first case, i.e. solution with anode sheath, we should not that the problem of the near anode presheath was not addressed in detail. To fill this gap, in the present paper we present 2D parametric analyses of the near anode region.

\section{Plasma-wall transition}

It was shown (Ref. 7) that the presheath scale length becomes comparable to the channel width under typical conditions of the Hall thruster channel. Thus, the model for the quasi-neutral plasma region is extended up to the sheath edge in order to provide the boundary condition at the plasmasheath interface as shown in Fig. 1.

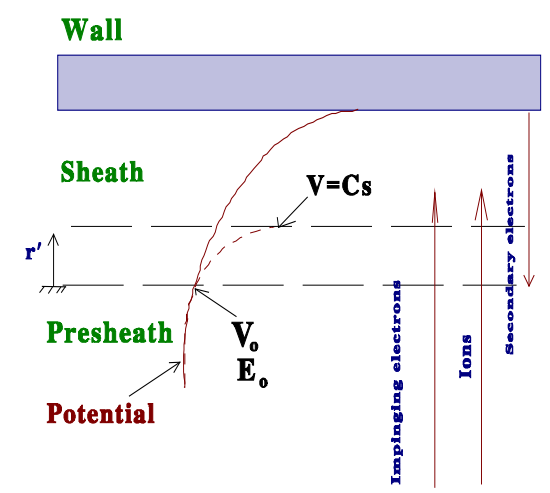

Figure 1. Schematic of the plasma-wall transition layer. The boundary at $r^{\prime}=0$ corresponds to smooth transition with monotonic potential behavior across the transition layer

The smooth transition between sheath and presheath is considered in the present work following the methodology developed previously by Beilis and Keidar. ${ }^{8}$ If the velocity at the sheath edge differs slightly from the Bohm velocity, the electric field becomes a continuous function. It was shown that a monotonic solution for the sheath problem could be obtained when the ion velocity at the sheath edge is smaller than the Bohm velocity ${ }^{8,12,13}$. In this case, the electric field becomes a 
continuous function increasing from a small non-zero value at the sheath edge up to a maximum value at the wall as shown schematically in Fig. 1. The solution criterion in that case is the minimal velocity at the presheath-sheath interface that results in a continuous solution for the potential distribution in the sheath ${ }^{8}$. Previously we applied this approach to the plasma-wall interaction in Hall thruster. It was shown that the ion velocity at the presheath edge increases with axial distance from about $0.7 \mathrm{Cs}$ up to Cs. This boundary velocity depends also on the secondary electron emission coefficient as shown in Fig. 2.
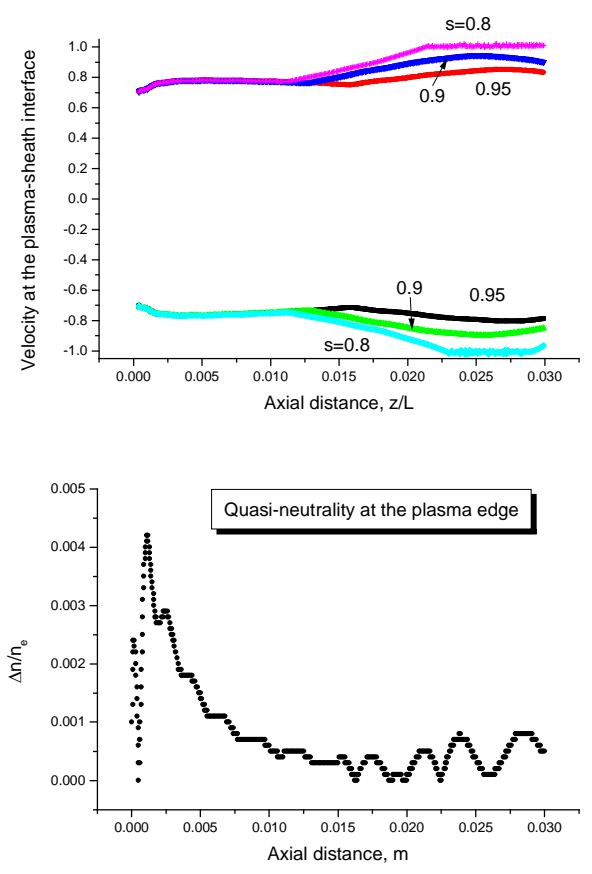

Figure 2.

Upper graph. The radial component of the plasma velocity at the plasma-sheath interface (normalized by the Bohm velocity) along the centerline with the secondary electron emission coefficient $s$ as a parameter. The negative velocity corresponds to the inner wall of the channel $\left(r=R_{I}\right)$ and the positive velocity corresponds to the outer wall of the channel $\left(r=R_{2}\right)$

Lower graph. Assessment of the quasi-neutrality condition along the channel length

It is appropriate to consider the applicability of this approach. It was pointed out in Ref. 7 that

when the velocity is small at the presheath edge, the electric field approaches to the value of $\frac{k T_{e}}{e L_{D}}$

where $T_{e}$ is the electron temperature and $L_{D}$ is the Debye length. It should be noted however that 
calculations for typical Hall thruster conditions show that electric field is usually much smaller than this limited value. One can argue that when the electric field approaches such a large value, the quasi-neutrality conditions explicitly assumed in the presheath equations may break down and therefore solution may be inaccurate. In order to check the validity of our approach under Hall thruster conditions, we calculate the deviation from quasi-neutrality and plot these results in Fig. 2 (lower graph). One can see that in the entire channel, the deviation from quasi-neutrality is lower than 0.005 , which can be considered negligible. Therefore, the approach that accounts for a nonzero electric field $E_{o}$ at the presheath-sheath interface in combination with the plasma velocity at that interface $V_{o}$ can be used for plasma-wall transition analyses in the Hall thruster.

\section{The model of near anode plasma region}

The anode presheath model is based on the assumption that the quasi-neutral region length (i.e. channel width $R_{2}-R_{1}$, see Fig. 3) is much larger than the Debye radius and therefore we will assume that $\mathrm{Z}_{\mathrm{i}} \mathrm{n}_{\mathrm{i}}=\mathrm{n}_{\mathrm{e}}=\mathrm{n}$, where $\mathrm{Z}_{\mathrm{i}}$ is the ion mean charge, $\mathrm{n}_{\mathrm{i}}$ is the ion density and $\mathrm{n}_{\mathrm{e}}$ is the electron density. For simplicity only single charge ions are considered in this paper $\left(Z_{i}=1\right)$. We will consider the plasma flow in an annular channel as shown in Fig. 3. A magnetic field with only a radial component, $\mathrm{B}_{\mathrm{r}}=\mathrm{B}$, is imposed. Cylindrical coordinates will be used, as shown in Fig. 3, with angle $\theta$, radius $\mathrm{r}$, and axial distance from the anode $\mathrm{z}$, respectively. The plasma flow starts in the near anode region and has lateral boundaries near the dielectric wall. The plasma presheath-sheath interface is considered to be the lateral boundary for the plasma flow region. A plasma will be considered with 'magnetized' electrons and 'unmagnetized' ions, i.e. $\rho_{\mathrm{e}}<<\mathrm{L}<<\rho_{\mathrm{i}}$, where $\rho_{\mathrm{e}}$ and $\rho_{\mathrm{i}}$ are the Larmor radii for the electrons and ions respectively, and $\mathrm{L}$ is the channel length. We employ a hydrodynamic model assuming: (i) the system reaches a steady state, and (ii) the electron component is not inertial, i.e. $\left(\mathbf{V}_{\mathrm{e}} \nabla\right) \bullet \mathbf{V}_{\mathrm{e}}=0$. 


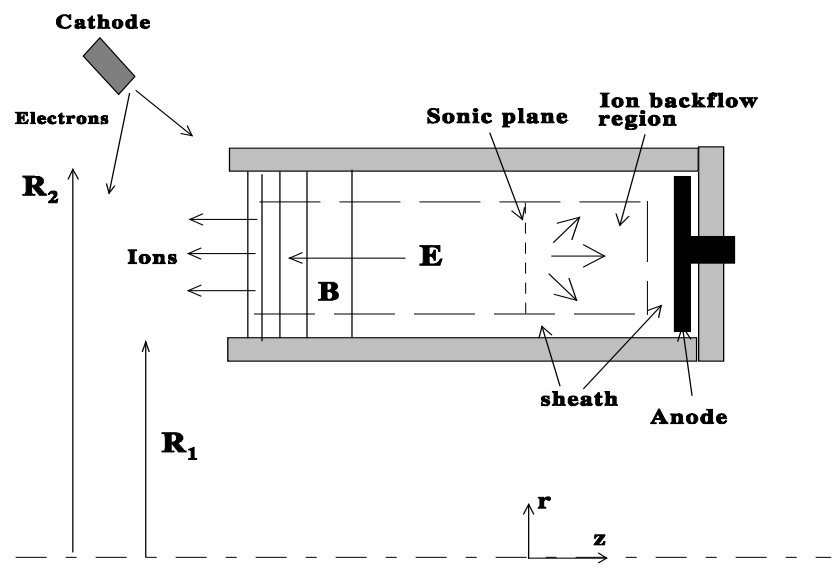

Figure 3. Schematic drawing the Hall thruster channel with presheath, sheath and ion backflow regions (not to scale)

The following system of equations describes the quasi-neutral plasma:

$$
\begin{aligned}
& n m_{i}\left(\mathbf{V}_{\mathrm{i}} \nabla\right) \mathbf{V}_{\mathrm{i}}=n e \mathbf{E}-\nabla \mathrm{P}_{\mathrm{i}}-\beta n m_{\mathrm{i}} \mathrm{n}_{\mathrm{a}}\left(\mathbf{V}_{\mathrm{i}}-\mathbf{V}_{\mathrm{a}}\right)+\mathrm{n} v_{\mathrm{m}} \mathrm{m}_{\mathrm{e}}\left(\mathbf{V}_{\mathrm{e}}-\mathbf{V}_{\mathrm{i}}\right) \\
& \nabla \cdot\left(\mathbf{V}_{\mathrm{i}} \mathrm{n}\right)=\beta \mathrm{nn}_{\mathrm{a}} \\
& \nabla \cdot\left(\mathbf{V}_{\mathrm{a}} \mathrm{n}_{\mathrm{a}}\right)=-\beta \mathrm{nn}_{\mathrm{a}} \\
& 0=-\operatorname{en}(\mathbf{E}+\mathbf{V} \times \mathbf{B})-\nabla P_{e}-n v_{m} m_{e}\left(\mathbf{V}_{e}-\mathbf{V}_{\mathbf{i}}\right)
\end{aligned}
$$

where $\mathrm{n}$ is the plasma density, $\beta$ is the ionization rate, $\mathrm{E}$ is the axial component of the electric field, $v_{\mathrm{m}}$ is the frequency of electron collisions. To simplify the problem, without missing the major physical effects, we consider one-dimensional flow of the neutrals. Previous analyses ${ }^{7}$ showed that the electron temperature is fairly uniform in the near anode region. Therefore we assume here that $\mathrm{T}_{\mathrm{e}}=$ const. The equation for the current density across the magnetic field can be obtained from Eq. 4 and has the form: 


$$
j_{z}=\frac{e n \mu_{e o}}{1+\left(\frac{\omega_{e}}{v_{m}}\right)^{2}}\left(E_{z}+\frac{k T_{e}}{e} \cdot \frac{\partial \ln n}{\partial z}\right)
$$

The equations for the heavy particles (ions and neutrals) may be written in component form in cylindrical coordinates. After some transformations, the equation system has the following form:

$\frac{\partial\left(n V_{z}\right)}{\partial z}+\frac{\partial\left(n V_{r}\right)}{\partial r}+\frac{n V_{r}}{r}=\beta n_{i} n_{a}$

$$
\frac{V_{z}^{2}-C_{s}^{2}}{V_{z}} \cdot \frac{\partial V_{z}}{\partial z}=j_{z} \frac{1+\left(\frac{\omega_{e}}{v_{m}}\right)^{2}}{m_{i} n \mu_{e o}}-V_{r} \frac{\partial V_{z}}{\partial r}-n_{a} V_{a} \beta-\frac{C_{s}^{2}}{V_{z}} \beta n_{a}
$$

$$
+\frac{V_{r}}{r V_{z}}+\frac{1}{n V_{z}} \frac{\partial\left(n V_{r}\right)}{\partial r}
$$

$V_{z} \frac{\partial V_{r}}{\partial z}=-V_{r} \frac{\partial V_{r}}{\partial r}-\frac{k T_{e}}{m_{i}} \cdot \frac{\partial \ln n}{\partial r}$

$\frac{\partial\left(n_{a} V_{a}\right)}{\partial z}=-\beta n_{i} n_{a}$

In this model, the electron flow will be considered separately along and across magnetic field lines. Due to the configuration of the magnetic field (i.e. only the radial magnetic field component is considered), the electron transport is greater in the azimuthal direction (E×B drift) than in the axial direction (drift diffusion due to collisions). According to Eq. 4, the electron transport equation along the magnetic field can be written as a balance between pressure and electric forces assuming that the current component in the radial direction is zero. If we assume that the electron temperature is constant along each magnetic field line we obtain that

$\varphi-\frac{k T_{e}}{e} \ln n=$ const 
This equation makes it possible to reduce the two-dimensional calculation of the electric field to a one-dimensional problem.

The total electron collision frequency considered in the present model consists of electron-neutral collisions and electron-ion Coulomb collisions, $v_{\mathrm{m}}=v_{\mathrm{en}}+v_{\mathrm{ei}}$. It should be noted that including only the classical mechanism of collisions cannot explain the electron transport observed in a Hall thruster channel. Among non-classical mechanisms some suggest that electron collisions with the walls play a major role ${ }^{14}$ while others obtained reasonable solutions including oscillations by assuming only anomalous transport ${ }^{15}$. In our Hall thruster channel model we took into account both effects $^{7}$. In the present work we will however consider only the classical mechanisms. The reason for this is that the magnetic field is relatively small near the anode and thus one does not expect Bohm type of transport associated with turbulence. Electron temperature is rather low in this region and thus electron-wall interaction is not expected to be significant. Additionally, recent estimations of the Hall parameter from experimental data show that this seems to be the case in the near anode region ${ }^{16}$. The electron-neutral collision frequency may be estimated as follows:

$v_{\mathrm{en}}=\mathrm{n}_{\mathrm{a}} \sigma_{\mathrm{ea}} \mathrm{V}_{\mathrm{th}}^{\mathrm{e}}$

where $\mathrm{n}_{\mathrm{a}}$ is the neutral density, $\sigma_{\mathrm{ea}}$ is the collision cross section dependent on the electron energy $\left(\sigma_{\text {ea }} \sim(10 \div 40) \times 10^{-20} \mathrm{~m}^{-2}\right.$ for Xenon, in considered energy range $)$, and $\mathrm{V}_{\text {th }}^{\mathrm{e}}$ is the electron thermal velocity.

\section{Results}

In this section we present some results of calculation of the plasma flow in the near anode presheath. As a first approximation we assume that the electron temperature, the neutral density and the neutral velocity 
are constant in the near anode region. This assumption is based on our previous calculations. ${ }^{7}$ Presented results correspond to $\mathrm{T}_{\mathrm{e}}=5 \mathrm{eV}$. When it is not specified, the calculations correspond to a mass flow rate of $4.5 \mathrm{mg} / \mathrm{s}$, a magnetic field of $10^{-3} \mathrm{~T}$ and an initial density of 0.03 (normalized by the neutral density). The numerical analysis is similar to that developed previously ${ }^{17}$.

The calculation of the plasma density in the near anode region is shown in Fig. 4 .

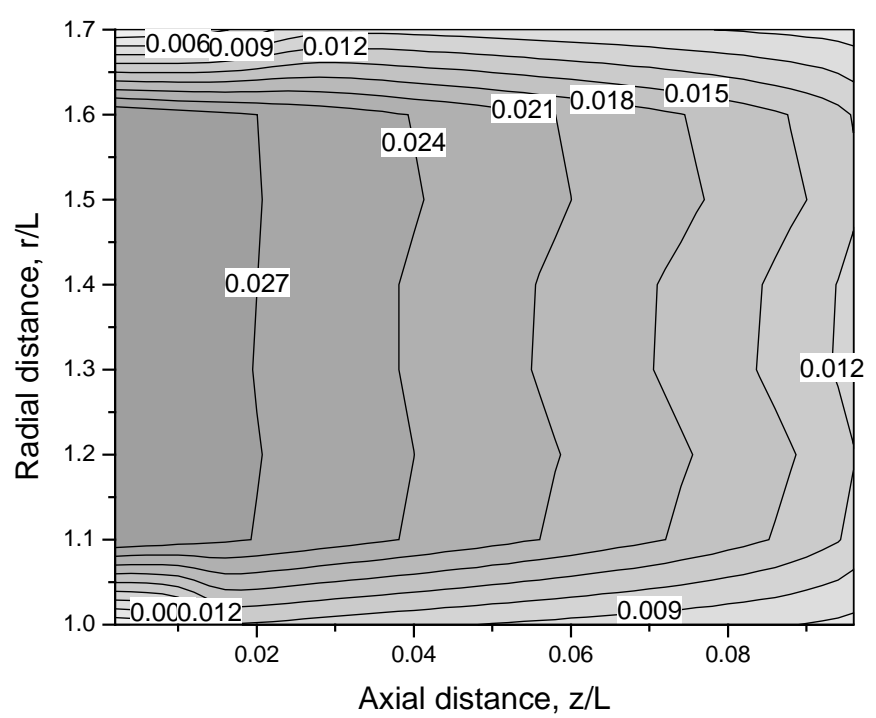

Figure 4. Plasma density distribution in the ion backflow region. $B=0.001$ T. Anode is located at the right side.

One can see that the plasma density in the near anode region is non-uniform. There is a strong gradient that develops toward the dielectric wall. Plasma density decreases toward the anode.

The velocity distribution (vector field) in the near anode presheath is shown in Fig. 5. It is clear from this figure that the anode presheath is essentially two-dimensional with ions accelerated toward the anode and toward the lateral dielectric wall. Only a narrow region along the channel centerline has a clear 1D structure of ions being accelerated toward the anode. When the magnetic field increases, the spatial extent of the anode presheath region decreases as shown in Fig. $5 \mathrm{~b}$. 


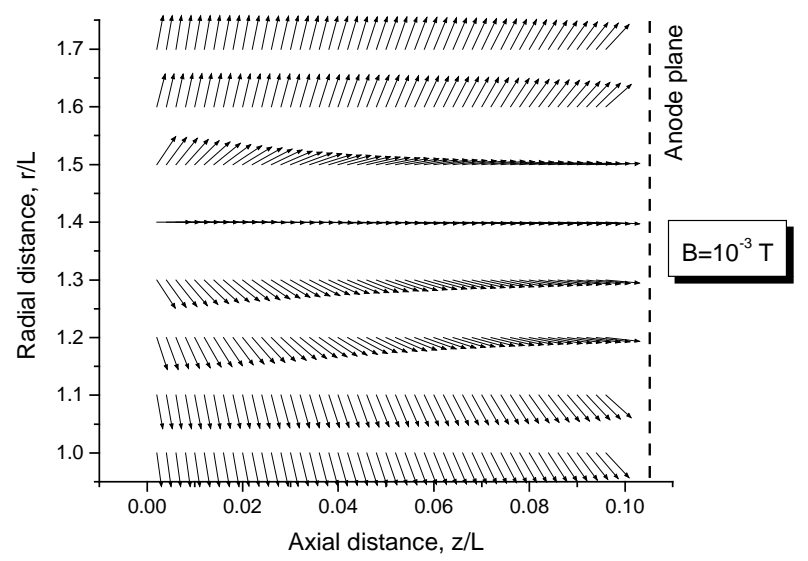

(a)

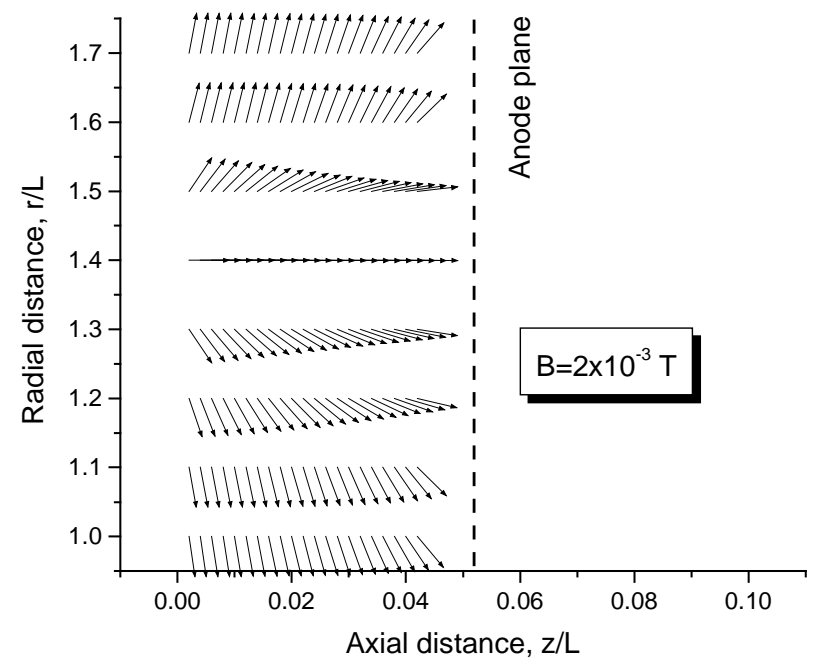

(b)

Figure 5. Ion velocity vector distribution in the near anode region.

The corresponding potential distribution is shown in Fig. 6. It can be seen that a much stronger electric field develops in the axial direction in the high magnetic field case. This is not surprising since the magnetic field plays a significant role in anode presheath formation. Essentially, the anode presheath can be called a magnetic presheath similar to one described previously ${ }^{18,19}$. 


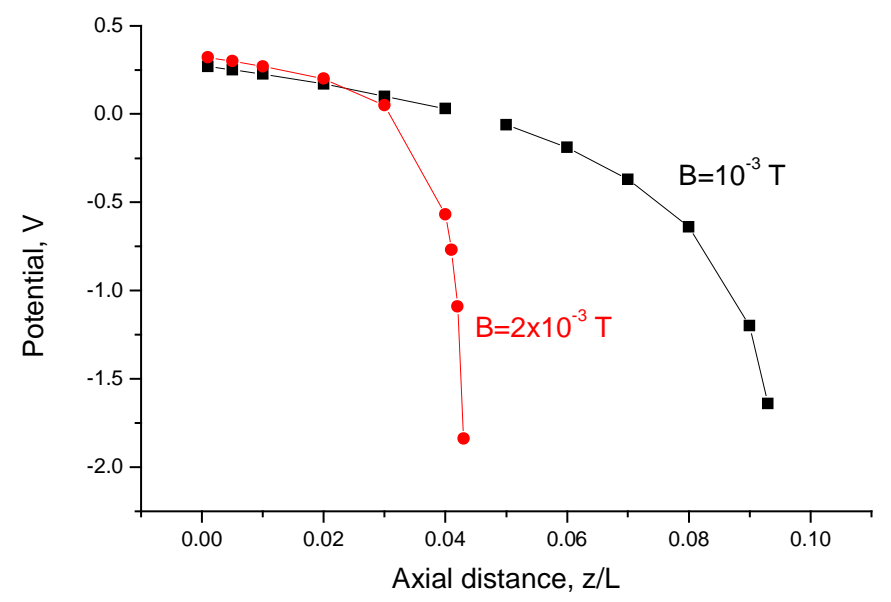

Figure 6. Potential distribution in the anode presheath with magnetic field as a parameter

An effect of the magnetic field on the anode presheath can be seen in Fig. 7, where presheath thickness and potential drop are shown. One can see that the anode presheath thickness decreases with magnetic field, according to the conventional wisdom of magnetic presheaths. The potential drop has nonmonotonic behavior with magnetic field. Initially it is negative and its magnitude increases, but at relatively high magnetic field potential changes sign and becomes positive.

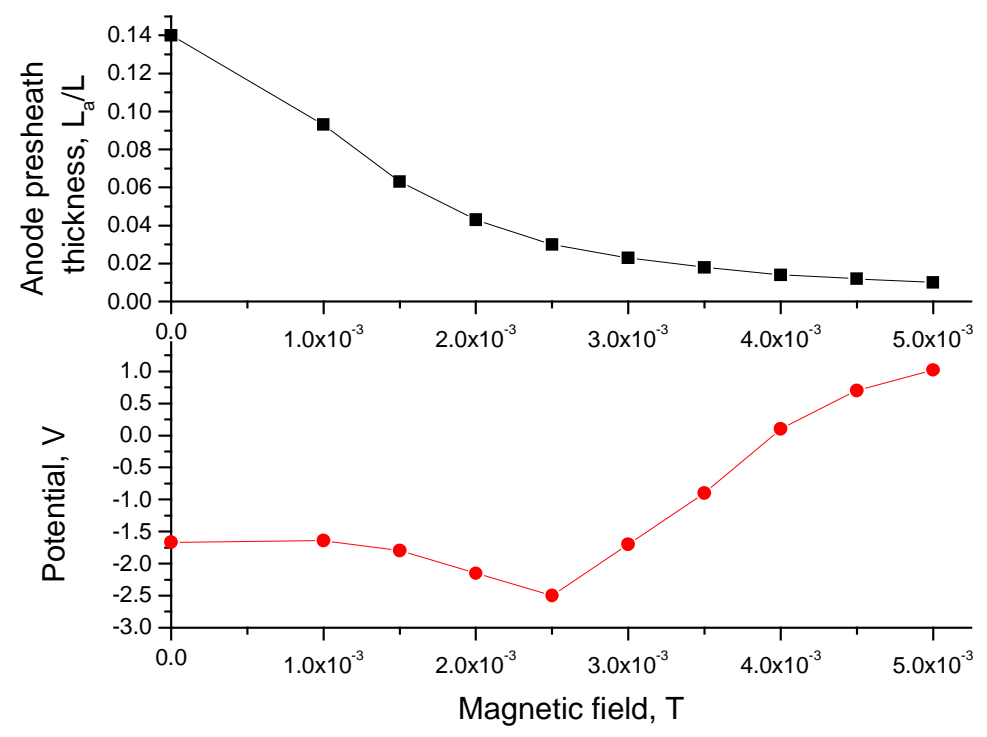

Figure 7. Anode presheath thickness and potential distribution as a function of a magnetic field 
Another interesting effect is presented in Fig. 8. It was shown that the plasma density is rather nonuniform in the Hall thruster channel (see for instance Ref. 7 and Fig. 4 in this paper). Therefore the initial density is really a function of radial position within the channel. Using a density distribution in the radial direction from previous calculations (Ref. 7) at a distance of $0.3 \mathrm{~cm}$ from the anode, we can calculate the anode presheath thickness. These results are shown in Fig. 8. One can see that the anode presheath thickness is significantly non-uniform in the radial direction. Therefore, in addition to a complicated 2D picture of the anode presheath presented in Fig. 5, one can expect that the anode presheath starting plane (and therefore the sonic boundary) will have a radial profile. This brings us to the conclusion that the sonic transition in the Hall thruster may have rather 2D structure.

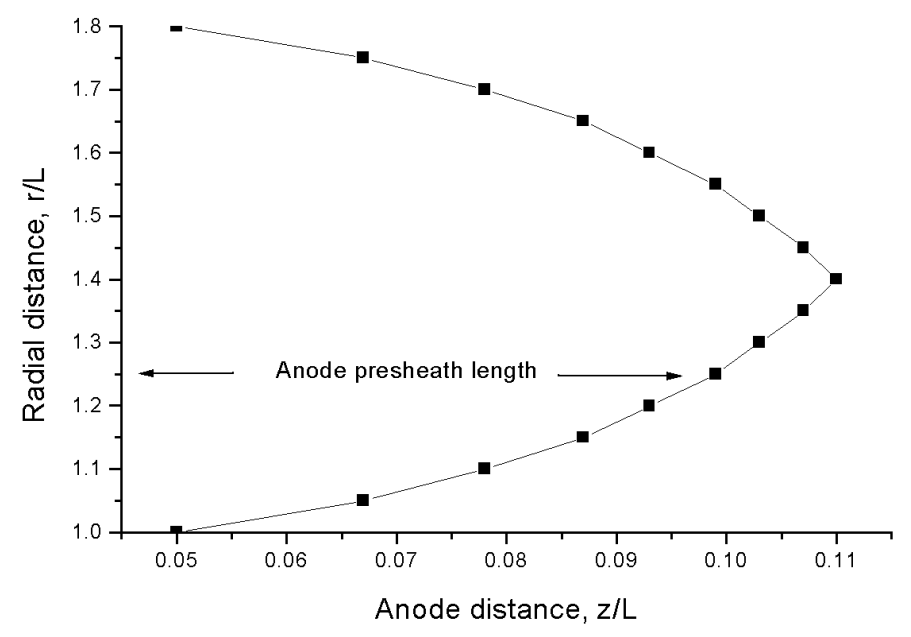

Figure 8. Anode presheath thickness in $r$-z plane

The dependence of the anode presheath thickness and potential distribution on the mass flow rate is shown in Fig. 9. It can be seen that anode presheath thickness initially increases with mass flow rate, reaches a maximum and then decreases. The reason for this is that at low flow rate (and therefore low neutral density) the magnetic presheath mechanism is realized and additional collision with neutrals decreases ion acceleration. In the high mass flow rate range, the presheath is rather collision-dominated 
and therefore neutral density increase leads to ion acceleration and as a result the presheath thickness decreases.
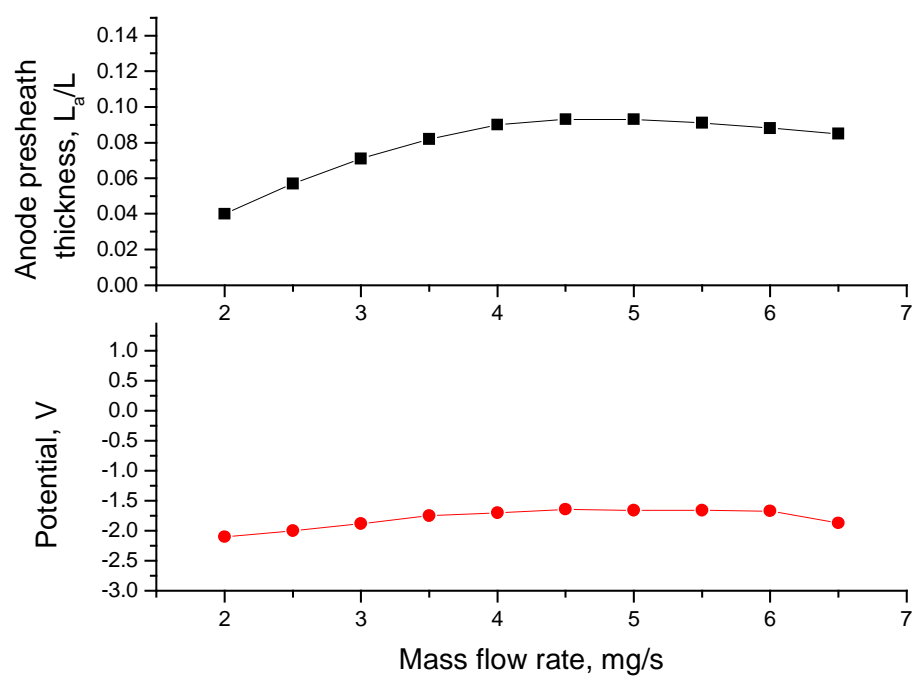

Figure 9. Anode presheath thickness and potential distribution as a function of a mass flow rate

\section{Concluding remarks}

In this paper, analyses of the anode region in the Hall thruster were presented. In the case when anode sheath existence is essential, the ion backflow region (presheath) was considered. It was shown that the anode presheath region has a 2D structure because two presheaths exist: one near the anode and one near the dielectric wall. Complex ion flow in the near anode region occurs in order to satisfy these requirements. It was shown that the length of the anode presheath is affected significantly by the magnetic field strength and the mass flow rate. It was concluded that the sonic transition in the Hall thruster may have a 2D structure. 


\section{REFERENCES}

${ }^{1}$ V. Kim, J. Prop. Power, 14, 736 (1998)

${ }^{2}$ V.V. Zhurin, H.R. Kaufman and R.S. Robinson, Plasma Sources Sci. Technol., 8, 1 (1999)

${ }^{3}$ A.I. Morozov and V.V. Savelyev, in Review of Plasma Physics, Ed. By B.B. Kadomtsev and V.D. Shafranov (Consultant Bureau, New York, 2000), Vol. 21, p. 203.

${ }^{4}$ E. Choueiri, Phys. Plasmas, 85025 (2001).

${ }^{5}$ Y. Raitses, J. Ashkenazy, G. Appelbaum and M. Guelman, $25^{\text {th }}$ Inter. Conference on Electric Propulsion, Cleveland, OH, (The Electric Rocket Propulsion Society, Worthington, OH, 1997) IEPC 97-056.

${ }^{6}$ Y. Raitses, L. Dorf, A. Litvak and N. Fisch, J. Appl. Phys., 88, 1263 (2000)

${ }^{7}$ M. Keidar, I.D. Boyd and I.I. Beilis, Phys. Plasmas, v. 8, No. 12, 5315 (2001).

${ }^{8}$ I. I. Beilis and M. Keidar, Phys. Plasmas 51545 (1998)

${ }^{9}$ A. Fruchtman, N.J. Fisch, J. Ashkenazy, and Y. Raitses, $25^{\text {th }}$ Inter. Conference on Electric Propulsion, Cleveland OH, 1997 (The Electric Rocket Propulsion Society, Cleveland OH) IEPC Paper No. 97-022.

${ }^{10}$ E. Ahedo, P. Martinez-Cerezo, and M. Martinez-Sanches, Phys. Plasmas 8, 3058 (2001)

${ }^{11}$ L. Dorf, V. Semenov, Y. Raitses, and N.J. Fisch, "The possibility of a Hall thruster operation in the absence of the anode sheath", Paper AIAA-2002-4246, and LANL preprint server, physics/0205034, Posted 11 May 2002, http://xxx.lanl.gov/ftp/physics/papers/0205/0205034.pdf

${ }^{12}$ I.I. Beilis, in book: I.I. Beilis, V.A. Bityurin, U.A. Vasiljeva, V.V. Kirillow, G.A. Lyubimov, S.A. Medin, A.E. Sheindlin, B.Ya. Shumjatsky, MHD energy conversion. Physical and technical aspects, edited by V.A. Kirillin and A.E. Sheyndlin, Moscow: Nauka, 1983, (368 pp.) English translation: (American Institute of Aeronautics and Astronautics, Inc., New York, 1986).

${ }^{13}$ V.A. Godyak and N. Sternberg, IEEE Trans. Plasma Sci., 18159 (1990).

${ }^{14}$ J. P. Bouef and L. Garrigues, J. Appl. Phys., 84, 3541 (1998).

${ }^{15}$ J. M. Fife and M. Martinez-Sanchez, $24^{\text {th }}$ Inter. Conference on Electric Propulsion, Moscow, Russia, 1995 (The Electric Rocket Propulsion Society, Worthington, OH, 1995) IEPC-95-240.

${ }^{16}$ N. B. Meezan, W.A. Hargus, and M. A. Cappelli, Phys. Rev., E63 026410 (2001)

${ }^{17}$ M. Keidar, I. Beilis, R L. Boxman and S. Goldsmith, J. Phys. D: Appl. Phys., 29, 1973 (1996).

${ }^{18}$ M. Keidar, I. Beilis, R. L. Boxman, and S. Goldsmith Proceeding XXII ICPIG (Stevens Institute of Technology, Hoboken, NJ, 1995), vol. 2 p. 157.

${ }^{19}$ I. I. Beilis, M. Keidar, and S. Goldsmith Phys. Plasmas 4(10) 3461 (1997). 